\title{
Multicloning and Multibroadcasting in Operator Algebras
}

\author{
Krzysztof Kaniowski $^{1} \cdot$ Katarzyna Lubnauer $^{1}$. \\ Andrzej Luczak ${ }^{1}$
}

Received: 4 November 2014 / Accepted: 23 March 2015 / Published online: 12 April 2015

(C) The Author(s) 2015. This article is published with open access at Springerlink.com

\begin{abstract}
We investigate multicloning and multibroadcasting in the general operator algebra framework in arbitrary dimension, generalizing thus results obtained in this framework for simple cloning and broadcasting.
\end{abstract}

Keywords Quantum states · Multicloning · Multibroadcasting · Quantum channels

\section{Introduction}

The celebrated no-cloning theorem for quantum states, which first appeared in [6, 14], has since been generalized in several directions, including also the no-broadcasting variant. Some of these generalizations concern also the investigations of the cases in which the states to be cloned (broadcast), as well as their clones or broadcast images, are states on tensor products algebras. Operations employed in these cases bear often the name of superbroadcasting; we shall use for them the names of multicloning and multibroadcasting.

Among numerous works in the area of cloning and broadcasting, let us mention $[3,10]$ in which the analysis is performed in the Hilbert space setup, $[1,2]$ where the setup is generic probabilistic models, and $[4,5,12]$ where the multi-fold variant is considered. A common

Work supported by NCN grant no 2011/01/B/ST1/03994

Krzysztof Kaniowski

kanio@math.uni.lodz.pl

$\triangle$ Katarzyna Lubnauer

lubnauer@math.uni.lodz.pl

Andrzej Łuczak

anluczak@math.uni.lodz.pl

1 Faculty of Mathematics and Computer Science, Łódź University, ul. S. Banacha 22, 90-238 Łódź, Poland 
feature of these approaches consists in restricting attention to the finite-dimensional models; moreover, in the Hilbert space setup the map defining cloning or broadcasting is assumed to be completely positive.

Cloning and broadcasting in the general operator algebra framework, i.e. the setup where instead of the full algebra of all bounded operators on a finite-dimensional Hilbert space an arbitrary von Neumann algebra is considered, was investigated in [7], where also the assumption of complete positivity of the broadcasting (cloning) operation was weakened to the form of the so-called $1 \frac{1}{2}$-positivity, i.e. the operation being a Schwarz (or strongly positive) map. The present paper aims at a generalization of some results from [7] to the multi-fold variant.

It is probably worth mentioning that for cloning alone the assumption of $1 \frac{1}{2}$ positivity can further be weakened to the case where the operation in question is merely positive (see [11]). However, we refrain from doing it in this paper, because having as the multibroadcasting and multiclonig operations Schwarz maps allows us to use the results on multibroadcasting also in the case of multicloning, thus making the paper more homogeneous. Another interesting problem is multicloning in $C^{*}$-algebras. The case of simple cloning was considered in [8], and it seems possible to generalize those results to the case of multicloning, but again for the sake of homogeneity this question is not considered in our paper.

In general, the operation of multibroadcasting states can be described as follows. For a state $\rho$ on an algebra $\mathcal{A}$, we consider its map to a larger algebra containing $\mathcal{A}$ as 'marginals' in such a way that after projecting the mapped state onto the marginals we obtain again the state $\rho$. Thus this larger algebra has the form $\mathcal{A} \otimes \mathcal{B} \otimes \mathcal{A}$ with some algebra $\mathcal{B}$, and the algebra $\mathcal{A}$ as the left and right marginal. Now, if $\tilde{\rho}$ is the mapped (三 broadcast) state on the algebra $\mathcal{A} \otimes \mathcal{B} \otimes \mathcal{A}$, then we should have

$$
\Pi_{1} \widetilde{\rho}=\rho=\Pi_{2} \widetilde{\rho},
$$

where $\Pi_{1}, \Pi_{2}$ are projections of states on $\mathcal{A} \otimes \mathcal{B} \otimes \mathcal{A}$ to the states on the left and right marginals, respectively, defined as

$$
\left(\Pi_{1} \widetilde{\rho}\right)(a)=\widetilde{\rho}\left(a \otimes \mathbb{1}_{\mathcal{B}} \otimes \mathbb{1}_{\mathcal{A}}\right), \quad\left(\Pi_{2} \widetilde{\rho}\right)(a)=\widetilde{\rho}\left(\mathbb{1}_{\mathcal{A}} \otimes \mathbb{1}_{\mathcal{B}} \otimes a\right), \quad a \in \mathcal{A} .
$$

This setup describes various modes of broadcasting, namely, if $\mathcal{B}$ is the complex numbers, we obtain the customary broadcasting from $\mathcal{A}$ to $\mathcal{A} \otimes \mathcal{A}$, while if $\mathcal{A}$ is of the form $\underbrace{\mathcal{C} \otimes \cdots \otimes \mathcal{C}}_{m \text { times }}$ and $\mathcal{B}=\underbrace{\mathcal{C} \otimes \cdots \otimes \mathcal{C}}_{n-2 m \text { times }}$, then we get $m$-to- $n$ multibroadcasting; in particular, for $m=1$ we have multibroadcasting from $\mathcal{A}$ to $\mathcal{A} \otimes \cdots \otimes \mathcal{A}$. However, in this last case a more natural definition seems to be as follows. Namely, we can define projections $\Pi_{i}, i=1, \ldots, n$, from states on $\mathcal{A} \otimes \cdots \otimes \mathcal{A}$ to states on $\mathcal{A}$ by the formula

$$
\left(\Pi_{i} \widetilde{\rho}\right)(a)=\tilde{\rho}\left(\mathbb{1}_{\mathcal{A}} \otimes \cdots \otimes \mathbb{1}_{\mathcal{A}} \otimes a_{i} \otimes \mathbb{1}_{\mathcal{A}} \otimes \cdots \otimes \mathbb{1}_{\mathcal{A}}\right), \quad a \in \mathcal{A},
$$

and require that

$$
\Pi_{i} \tilde{\rho}=\rho \quad \text { for each } \quad i=1, \ldots, n,
$$

while the former definition requires only that

$$
\Pi_{1} \widetilde{\rho}=\Pi_{n} \tilde{\rho}=\rho .
$$

It comes rather unexpectedly that the seemingly stronger condition (1) of multibroadcastability is in fact equivalent to the same formula holding just for arbitrary two fixed indices $j$ and $k$, i.e. the equality

$$
\Pi_{j} \tilde{\rho}=\Pi_{k} \tilde{\rho}
$$


As for multicloning, it consists in mapping a state $\rho$ to the state $\rho \otimes \cdots \otimes \rho$, so in the scheme above we should have $\mathcal{B}=\underbrace{\mathcal{A} \otimes \cdots \otimes \mathcal{A}}_{n-2 \text { times }}$ for some $n \geq 2$.

The main results of this work are as follows. As algebras $\mathcal{A}$ and $\mathcal{B}$ in the above scheme we take von Neumann algebras with the tensor product $\mathcal{A} \bar{\otimes} \mathcal{B} \bar{\otimes} \mathcal{A}$, and all the states considered will be normal, i.e. belonging to respective preduals. For an arbitrary subset $\Gamma$ of the normal states of $\mathcal{A}$, it is shown that $\Gamma$ is multibroadcastable if and only if there is a countable family of normal states having mutually orthogonal supports such that each $\rho \in \Gamma$ is a convex combination of these states, generalizing thus a result obtained in [7] for the case of simple broadcasting. Moreover, it is shown that $\Gamma$ is multicloneable if and only if the states from it have mutually orthogonal supports, which again generalizes a similar result in [7].

It should be noted that in this work we follow in many respects the basic ideas of [7], exploiting them in the multi-fold case.

\section{Preliminaries and Notation}

Let $\mathcal{A}$ be an arbitrary von Neumann algebra with identity $\mathbb{1}_{\mathcal{A}}$ acting on a Hilbert space $\mathcal{H}$. The predual $\mathcal{A}_{*}$ of $\mathcal{A}$ is a Banach space of all normal, i.e. continuous in the $\sigma$-weak topology, linear functionals on $\mathcal{A}$.

A state on $\mathcal{A}$ is a bounded positive linear functional $\rho: \mathcal{A} \rightarrow \mathbb{C}$ of norm one. For a normal state $\rho$ its support, denoted by $\mathrm{s}(\rho)$, is defined as the smallest projection in $\mathcal{A}$ such that $\rho(\mathrm{s}(\rho))=\rho\left(\mathbb{1}_{\mathcal{A}}\right)$. In particular, we have

$$
\rho(\mathrm{s}(\rho) a)=\rho(a \mathrm{~s}(\rho))=\rho(a), \quad a \in \mathcal{A},
$$

and if $\rho(\mathrm{s}(\rho) a \mathrm{~s}(\rho))=0$ for $\mathrm{s}(\rho) a \mathrm{~s}(\rho) \geq 0$, then $\mathrm{s}(\rho) a \mathrm{~s}(\rho)=0$.

A normal state $\rho$ is said to be faithful if for each positive element $a \in \mathcal{A}$, from the equality $\rho(a)=0$ it follows that $a=0$. It is easily seen that the faithfulness of $\rho$ is equivalent to the relation $\mathrm{s}(\rho)=\mathbb{1}_{\mathcal{A}}$.

Let $\Gamma$ be a family of normal states on a von Neumann algebra $\mathcal{A}$. Define the support of this family by

$$
e=\bigvee_{\rho \in \Gamma} \mathrm{s}(\rho) .
$$

The family $\Gamma$ is said to be faithful if for each positive element $a \in \mathcal{A}$ from the equality $\rho(a)=0$ for all $\rho \in \Gamma$ it follows that $a=0$. Similarly to the case of one state it is seen that the faithfulness of this family is equivalent to the relation $e=\mathbb{1}_{\mathcal{A}}$; moreover, $\Gamma$ is faithful on the $W^{*}$-algebra $e \mathcal{A} e$.

By a $W^{*}$-algebra of operators acting on a Hilbert space $\mathcal{H}$ we shall mean a $C^{*}$-subalgebra of $\mathrm{B}(\mathcal{H})$ with identity, closed in the weak-operator topology.

A projection in a $W^{*}$-algebra is said to be minimal if it majorizes no other nonzero projection in this algebra. A $W^{*}$-algebra is said to be atomic if the supremum of all its minimal projections equals the identity of this algebra.

Let $\mathcal{A}$ and $\mathcal{B}$ be $W^{*}$-algebras. A linear map $T: \mathcal{A} \rightarrow \mathcal{B}$ is said to be normal if it is continuous in the $\sigma$-weak topologies on $\mathcal{A}$ and $\mathcal{B}$, respectively. It is called unital if it maps the unit of $\mathcal{A}$ to the unit of $\mathcal{B}$. The map $T$ is said to be Schwarz (or $1 \frac{1}{2}$-positive or strongly positive) if for each $a \in \mathcal{A}$ the following Schwarz inequality holds

$$
T(a)^{*} T(a) \leq\|T\| T\left(a^{*} a\right),
$$


which for a unital map amounts simply to

$$
T(a)^{*} T(a) \leq T\left(a^{*} a\right) .
$$

In particular, a completely or two- positive map is Schwarz. For a normal unital Schwarz map $T$ we define its multiplicative domain as

$$
\mathcal{N}=\left\{a \in \mathcal{A}: T\left(a^{*} a\right)=T(a)^{*} T(a), T\left(a a^{*}\right)=T(a) T(a)^{*}\right\} .
$$

It is known that $\mathcal{N}$ is a $W^{*}$-subalgebra of $\mathcal{A}$, and $T \mid \mathcal{N}$ is a *-homomorphism.

Let $\mathcal{A}$ and $\mathcal{B}$ be von Neumann algebras, and consider the tensor product $\mathcal{A} \bar{\otimes} \mathcal{B} \bar{\otimes} \mathcal{A}$. We have obvious counterparts

$$
\Pi_{1}, \Pi_{2}:(\mathcal{A} \bar{\otimes} \mathcal{B} \bar{\otimes} \mathcal{A})_{*} \rightarrow \mathcal{A}_{*}
$$

of the customary notion of partial trace employed in the case $\mathcal{A}=\mathrm{B}(\mathcal{H})$, defined as

$$
\left(\Pi_{1} \widetilde{\rho}\right)(a)=\widetilde{\rho}\left(a \otimes \mathbb{1}_{\mathcal{B}} \otimes \mathbb{1}_{\mathcal{A}}\right), \quad\left(\Pi_{2} \widetilde{\rho}\right)(a)=\widetilde{\rho}\left(\mathbb{1}_{\mathcal{A}} \otimes \mathbb{1}_{\mathcal{B}} \otimes a\right),
$$

for $\tilde{\rho} \in(\mathcal{A} \bar{\otimes} \mathcal{B} \bar{\otimes} \mathcal{A})_{*}$, and $a \in \mathcal{A}$. (The situation when $\mathcal{B}=\underbrace{\mathcal{A} \otimes \ldots \mathcal{A}}_{n-2 \text { times }}$, and we can define projections $\Pi_{i}$ for $i=1, \ldots, n$, as in the Introduction will be commented on later.)

The main objects of our interest are the following two operations of multibroadcasting and multicloning of states.

A linear map $K_{*}: \mathcal{A}_{*} \rightarrow(\mathcal{A} \bar{\otimes} \mathcal{B} \bar{\otimes} \mathcal{A})_{*}$ sending states to states and such that its dual $K: \mathcal{A} \bar{\otimes} \bar{\otimes} \mathcal{A} \rightarrow \mathcal{A}$ is a unital Schwarz map will be called a channel. (This terminology is almost standard, because by a 'channel' is usually meant a completely positive unital map between two von Neumann algebras.) A state $\rho \in \mathcal{A}_{*}$ is multibroadcast by channel $K_{*}$ if $\left(\Pi_{i} \circ K_{*}\right)(\rho)=\rho, i=1,2$; in other words, $\rho$ is multibroadcast by $K_{*}$ if for each $a \in \mathcal{A}$

$$
\rho\left(K\left(a \otimes \mathbb{1}_{\mathcal{B}} \otimes \mathbb{1}_{\mathcal{A}}\right)\right)=\rho\left(K\left(\mathbb{1}_{\mathcal{A}} \otimes \mathbb{1}_{\mathcal{B}} \otimes a\right)\right)=\rho(a) .
$$

A family of states is said to be multibroadcastable if there is a channel $K_{*}$ that multibroadcasts each member of this family.

Let $\mathcal{B}=\underbrace{\mathcal{A} \bar{\otimes} \ldots \bar{\otimes} \mathcal{A}}_{n-2 \text { times }}$. A state $\rho \in \mathcal{A}_{*}$ is multicloned by channel $K_{*}$ if $K_{*} \rho=$ $\underbrace{\rho \otimes \cdots \otimes \rho}_{n \text { times }}$. A family of states is said to be multicloneable if there is a channel $K_{*}$ that multiclones each member of this family.

\section{Multibroadcasting}

In this section, we aim at a description of an arbitrary set of multibroadcastable states, thus let $\Gamma \subset \mathcal{A}_{*}$ be such. Then there is a channel $K_{*}$ which multibroadcasts the states in $\Gamma$. Define maps $L, R: \mathcal{A} \rightarrow \mathcal{A}$ as

$$
L(a)=K\left(a \otimes \mathbb{1}_{\mathcal{B}} \otimes \mathbb{1}_{\mathcal{A}}\right), \quad R(a)=K\left(\mathbb{1}_{\mathcal{A}} \otimes \mathbb{1}_{\mathcal{B}} \otimes a\right), \quad a \in \mathcal{A} .
$$

Then $L$ and $R$ are unital normal Schwarz maps on $\mathcal{A}$. Observe that for a state $\rho$ broadcast by $K_{*}$ we have, for each $a \in \mathcal{A}$,

$$
\begin{aligned}
& (\rho \circ L)(a)=\rho\left(K\left(a \otimes \mathbb{1}_{\mathcal{A}} \otimes \mathbb{1}_{\mathcal{B}}\right)\right)=\rho(a), \\
& (\rho \circ R)(a)=\rho\left(K\left(\mathbb{1}_{\mathcal{A}} \otimes \mathbb{1}_{\mathcal{B}} \otimes a\right)\right)=\rho(a),
\end{aligned}
$$

i.e.

$$
\rho \circ L=\rho \circ R=\rho,
$$


and by the very definition of multibroadcastability if (2) holds, then $\rho$ is multibroadcast by $K_{*}$. Consequently,

$$
\Gamma \subset\{\rho-\text { normal state }: \rho \circ L=\rho \circ R=\rho\} .
$$

To simplify our further considerations, we assume that $\Gamma$ is a faithful family of states. This assumption is not essential, as will be indicated later on.

For a map $T$ on $\mathcal{A}$, denote by $\mathcal{F}(T)$ its fixed-point space, i.e.

$$
\mathcal{F}(T)=\{a \in \mathcal{A}: T(a)=a\} .
$$

Let $\mathcal{N}$ be the multiplicative domain of $K$.

\section{Lemma 1 The following relations hold}

(i) for each $a \in \mathcal{F}(L)$ we have $a \otimes \mathbb{1}_{\mathcal{A}} \otimes \mathbb{1}_{\mathcal{B}} \in \mathcal{N}$,

(ii) for each $a \in \mathcal{F}(R)$ we have $\mathbb{1}_{\mathcal{A}} \otimes \mathbb{1}_{\mathcal{B}} \otimes a \in \mathcal{N}$.

Proof It is enough to prove (i) since the proof of (ii) is analogous. Let $a \in \mathcal{F}(L)$. The Schwarz inequality for the map $L$ yields

$$
a^{*} a=L(a)^{*} L(a) \leq L\left(a^{*} a\right),
$$

hence

$$
L\left(a^{*} a\right)-a^{*} a \geq 0 .
$$

For an arbitrary $\rho \in \Gamma$, we have on account of the $L$-invariance of $\rho$

$$
\rho\left(L\left(a^{*} a\right)-a^{*} a\right)=\rho\left(L\left(a^{*} a\right)\right)-\rho\left(a^{*} a\right)=0,
$$

and since $\Gamma$ is faithful we obtain

$$
L\left(a^{*} a\right)-a^{*} a=0,
$$

i.e.

$$
L\left(a^{*} a\right)=a^{*} a .
$$

Taking into account the definition of $L$, we get

$$
\begin{aligned}
& K\left(a^{*} \otimes \mathbb{1}_{\mathcal{B}} \otimes \mathbb{1}_{\mathcal{A}}\right) K\left(a \otimes \mathbb{1}_{\mathcal{B}} \otimes \mathbb{1}_{\mathcal{A}}\right)=L\left(a^{*}\right) L(a)=a^{*} a=L\left(a^{*} a\right) \\
& =K\left(a^{*} a \otimes \mathbb{1}_{\mathcal{B}} \otimes \mathbb{1}_{\mathcal{A}}\right)=K\left(\left(a^{*} \otimes \mathbb{1}_{\mathcal{B}} \otimes \mathbb{1}_{\mathcal{A}}\right)\left(a \otimes \mathbb{1}_{\mathcal{B}} \otimes \mathbb{1}_{\mathcal{A}}\right)\right),
\end{aligned}
$$

and changing $a$ to $a^{*}$ we find that

$$
K\left(a \otimes \mathbb{1}_{\mathcal{B}} \otimes \mathbb{1}_{\mathcal{A}}\right) K\left(a^{*} \otimes \mathbb{1}_{\mathcal{B}} \otimes \mathbb{1}_{\mathcal{A}}\right)=K\left(\left(a \otimes \mathbb{1}_{\mathcal{B}} \otimes \mathbb{1}_{\mathcal{A}}\right)\left(a^{*} \otimes \mathbb{1}_{\mathcal{B}} \otimes \mathbb{1}_{\mathcal{A}}\right)\right),
$$

showing that $a \otimes \mathbb{1}_{\mathcal{B}} \otimes \mathbb{1}_{\mathcal{A}}$ belongs to $\mathcal{N}$.

Let $\mathfrak{S}$ be the semigroup of normal Schwarz maps on $\mathcal{A}$ generated by $L$ and $R$. Denote by $\mathcal{F}(\mathfrak{S})$ the fixed-point space of $\mathfrak{S}$, i.e.

$$
\mathcal{F}(\mathfrak{S})=\{a \in \mathcal{A}: S(a)=a \text { for each } S \in \mathfrak{S}\} .
$$

The ergodic theorem for $W^{*}$-algebras proved in [9] yields that $\mathcal{F}(\mathfrak{S})$ is a von Neumann algebra, and there exists a normal faithful conditional expectation $\mathbb{E}: \mathcal{A} \rightarrow \mathcal{F}(\mathfrak{S})$ such that

$$
\mathbb{E} S=S \mathbb{E}=\mathbb{E}, \quad \text { for each } \quad S \in \mathfrak{S},
$$

and

$$
\rho \circ \mathbb{E}=\rho, \quad \text { for each } \rho \in \Gamma .
$$


Moreover, if $\varphi$ is an arbitrary $\mathbb{E}$-invariant normal state on $\mathcal{A}$, then from relation (3) we see that $\varphi$ is $\mathfrak{S}$-invariant. Conversely, if $\varphi$ is an arbitrary $\mathfrak{S}$-invariant normal state on $\mathcal{A}$, then another consequence of the ergodic theorem is that $\varphi$ is also $\mathbb{E}$-invariant (this follows from the fact that for each $a \in \mathcal{A}, \mathbb{E} a$ lies in the $\sigma$-weak closure of the convex hull of $\{S a: S \in \mathfrak{S}\})$. Consequently, we have the following equivalence for a normal state $\varphi$ on $\mathcal{A}$ :

$$
\varphi \text { is } \mathfrak{S} \text {-invariant if and only if it is } \mathbb{E} \text {-invariant. }
$$

It turns out that the map $K$ has a special form on the tensor product von Neumann algebra $\mathcal{F}(\mathfrak{S}) \bar{\otimes} \mathcal{B} \bar{\otimes} \mathcal{F}(\mathfrak{S})$.

Proposition 2 For each $x, y \in \mathcal{F}(\mathfrak{S})$, we have

$$
K\left(x \otimes \mathbb{1}_{\mathcal{B}} \otimes y\right)=x y ;
$$

moreover, the von Neumann algebra $\mathcal{F}(\mathfrak{S})$ is abelian.

Proof Considering the semigroups $\left\{L^{n}: n=0,1, \ldots\right\}$ and $\left\{R^{n}: n=0,1, \ldots\right\}$ generated by $L$ and $R$ we immediately notice that the fixed-point spaces of these semigroups are equal to $\mathcal{F}(L)$ and $\mathcal{F}(R)$, respectively, and the above-mentioned ergodic theorem shows that $\mathcal{F}(L)$ and $\mathcal{F}(R)$ are von Neumann algebras. Moreover,

$$
\mathcal{F}(\mathfrak{S})=\mathcal{F}(L) \cap \mathcal{F}(R) .
$$

Let $x, y \in \mathcal{F}(\mathfrak{S})$. Then by virtue of Lemma 1 we have

$$
x \otimes \mathbb{1}_{\mathcal{B}} \otimes \mathbb{1}_{\mathcal{A}} \in \mathcal{N} \quad \text { and } \quad \mathbb{1}_{\mathcal{A}} \otimes \mathbb{1}_{\mathcal{B}} \otimes y \in \mathcal{N},
$$

and thus from the homomorphic nature of $K$ on $\mathcal{N}$ we get

$$
\begin{aligned}
K\left(x \otimes \mathbb{1}_{\mathcal{B}} \otimes y\right) & =K\left(\left(x \otimes \mathbb{1}_{\mathcal{B}} \otimes \mathbb{1}_{\mathcal{A}}\right)\left(\mathbb{1}_{\mathcal{A}} \otimes \mathbb{1}_{\mathcal{B}} \otimes y\right)\right) \\
& =K\left(x \otimes \mathbb{1}_{\mathcal{B}} \otimes \mathbb{1}_{\mathcal{A}}\right) K\left(\mathbb{1}_{\mathcal{A}} \otimes \mathbb{1}_{\mathcal{B}} \otimes y\right)=L(x) R(y)=x y,
\end{aligned}
$$

and by the same token

$$
K\left(x \otimes \mathbb{1}_{\mathcal{B}} \otimes y\right)=K\left(\left(\mathbb{1}_{\mathcal{A}} \otimes \mathbb{1}_{\mathcal{B}} \otimes y\right)\left(x \otimes \mathbb{1}_{\mathcal{B}} \otimes \mathbb{1}_{\mathcal{A}}\right)\right)=R(y) L(x)=y x,
$$

showing the claim.

We have the following important property of a map satisfying Eq. 5.

Proposition 3 Let $\mathcal{M}$ be an abelian von Neumann algebra, and let $\mathcal{B}$ be an arbitrary von Neumann algebra. The following conditions are equivalent:

(i) there exists a bounded normal Schwarz map $\widetilde{K}: \mathcal{M} \bar{\otimes} \mathcal{B} \bar{\otimes} \mathcal{M} \rightarrow \mathcal{M}$ satisfying the relation

$$
\widetilde{K}\left(x \otimes \mathbb{1}_{\mathcal{B}} \otimes y\right)=x y, \quad x, y \in \mathcal{M},
$$

(ii) $\mathcal{M}$ is atomic, i.e. generated by the minimal projections.

Proof $(\mathrm{i}) \Longrightarrow$ (ii). Define a map $\widehat{K}: \mathcal{M} \bar{\otimes} \mathcal{M} \rightarrow \mathcal{M}$ by the formula

$$
\widehat{K}(x \otimes y)=\widetilde{K}\left(x \otimes \mathbb{1}_{\mathcal{B}} \otimes y\right), \quad x, y \in \mathcal{M} .
$$

Then $\widehat{K}$ is a bounded normal Schwarz map from $\mathcal{M} \bar{\otimes} \mathcal{M}$ to $\mathcal{M}$ satisfying the condition

$$
\widehat{K}(x \otimes y)=x y, \quad x, y \in \mathcal{M} .
$$

From [7, Proposition 6], it follows that $\mathcal{M}$ is atomic. 
(ii) $\Longrightarrow$ (i). Since $\mathcal{M}$ is an abelian von Neumann algebra generated by the minimal projections, it has the form

$$
\mathcal{M}=\left\{\sum_{i} \alpha_{i} e_{i}: \alpha_{i} \in \mathbb{C}, \sup _{i}\left|\alpha_{i}\right|<\infty\right\},
$$

where the $e_{i}$ are pairwise orthogonal minimal projections. Consequently, each $x \in \mathcal{M}$ is represented as

$$
x=\sum_{i} \varphi_{i}(x) e_{i}
$$

where $\varphi_{i}(x) \in \mathbb{C}$ are coefficients depending on $x$. Thus

$$
x e_{i}=\varphi_{i}(x) e_{i},
$$

which shows that the $\varphi_{i}$ are normal states on $\mathcal{M}$ with supports $e_{i}$. Pick a normal state $\psi$ on $\mathcal{B}$ and define a map $\widetilde{K}_{*}$ from $\mathcal{M}_{*}$ to $(\mathcal{M} \bar{\otimes} \mathcal{B} \bar{\otimes} \mathcal{M})_{*}$ by the formula

$$
\widetilde{K}_{*} \varphi=\sum_{i} \varphi\left(e_{i}\right) \varphi_{i} \otimes \psi \otimes \varphi_{i}, \quad \varphi \in \mathcal{M}_{*} .
$$

The series on the right hand side is clearly norm-convergent for $\varphi \in \mathcal{M}_{*}^{+}$, so it is normconvergent for all $\varphi \in \mathcal{M}_{*}$. Moreover, for $\varphi \in \mathcal{M}_{*}^{+}$we have

$$
\left\|\widetilde{K}_{*} \varphi\right\| \leq \sum_{i} \varphi\left(e_{i}\right)=\varphi(\mathbb{1})=\|\varphi\|,
$$

so $\widetilde{K}_{*}$ is bounded and maps $\mathcal{M}_{*}$ into $(\mathcal{M} \bar{\otimes} \mathcal{B} \bar{\otimes} \mathcal{M})_{*}$. Its adjoint $\widetilde{K}: \mathcal{M} \bar{\otimes} \mathcal{B} \bar{\otimes} \mathcal{M} \rightarrow \mathcal{M}$ is a bounded normal Schwarz map having the form

$$
\widetilde{K}(\tilde{x})=\sum_{i} \varphi_{i} \otimes \psi \otimes \varphi_{i}(\tilde{x}) e_{i}, \quad \tilde{x} \in \mathcal{M} \bar{\otimes} \mathcal{B} \bar{\otimes} \mathcal{M},
$$

so in particular,

$$
\tilde{K}\left(x \otimes \mathbb{1}_{\mathcal{B}} \otimes y\right)=\sum_{i} \varphi_{i}(x) \varphi_{i}(y) e_{i}, \quad x, y \in \mathcal{M} .
$$

On the other hand, we have

$$
x=\sum_{i} \varphi_{i}(x) e_{i}, \quad y=\sum_{i} \varphi_{i}(y) e_{i},
$$

hence

$$
x y=\sum_{i} \varphi_{i}(x) \varphi_{i}(y) e_{i},
$$

showing that

$$
\widetilde{K}\left(x \otimes \mathbb{1}_{\mathcal{B}} \otimes y\right)=x y,
$$

which ends the proof.

From Propositions 2 and 3, we obtain an important corollary.

Proposition 4 The algebra $\mathcal{F}(\mathfrak{S})$ is atomic and abelian.

We have the following characterization of an arbitrary set of multibroadcastable states.

Theorem 5 Let $\Gamma$ be an arbitrary faithful family of normal states on $\mathcal{A}$. The following conditions are equivalent 
(i) $\Gamma$ is multibroadcastable,

(ii) there exists a family $\left\{\omega_{i}\right\}$ of normal states with pairwise orthogonal supports such that each $\rho \in \Gamma$ is a (possibly infinite) convex combination of $\omega_{i}$.

Proof (i) $\Longrightarrow$ (ii). Let $K_{*}$ be a channel multibroadcasting the states in $\Gamma$. Applying to $K_{*}$ our previous considerations, we obtain that $\mathcal{F}(\mathfrak{S})$ is an abelian atomic von Neumann algebra. Now, the proof follows the lines of the proof of Theorem 3.10 in [7].

(ii) $\Longrightarrow$ (i). Assume that for each $\rho \in \Gamma$ we have

$$
\rho=\sum_{i} \lambda_{i} \omega_{i}
$$

where $\lambda_{i} \geq 0$,

$$
\sum_{i} \lambda_{i}=1
$$

and that the states $\omega_{i}$ have pairwise orthogonal supports $e_{i}$. Take an arbitrary normal state $\psi$ on $\mathcal{B}$ and define a channel $\widetilde{K}_{*}: \mathcal{A}_{*} \rightarrow(\mathcal{A} \bar{\otimes} \mathcal{B} \bar{\otimes} \mathcal{A})_{*}$ similarly as in Proposition 3, i.e.

$$
\widetilde{K}_{*} \varphi=\sum_{i} \varphi\left(e_{i}\right) \omega_{i} \otimes \psi \otimes \omega_{i}, \quad \varphi \in \mathcal{A}_{*}
$$

Its adjoint is a normal unital completely positive map from $\mathcal{A} \bar{\otimes} \mathcal{B} \bar{\otimes} \mathcal{A}$ into $\mathcal{A}$ having the form

$$
\widetilde{K}(\tilde{x})=\sum_{i} \omega_{i} \otimes \psi \otimes \omega_{i}(\tilde{x}) e_{i}, \quad \tilde{x} \in \mathcal{A} \bar{\otimes} \mathcal{B} \bar{\otimes} \mathcal{A}
$$

For $\rho$ given by (6), we have

$$
\begin{aligned}
\rho\left(\tilde{K}\left(a \otimes \mathbb{1}_{\mathcal{B}} \otimes \mathbb{1}_{\mathcal{A}}\right)\right) & =\sum_{j} \lambda_{j}\left(\sum_{i} \omega_{i}(a) \omega_{j}\left(e_{i}\right)\right)=\sum_{j} \lambda_{j} \omega_{j}(a) \omega_{j}\left(e_{j}\right) \\
& =\sum_{j} \lambda_{j} \omega_{j}(a)=\rho(a),
\end{aligned}
$$

and in the same way we get

$$
\rho\left(\widetilde{K}\left(\mathbb{1}_{\mathcal{A}} \otimes \mathbb{1}_{\mathcal{B}} \otimes a\right)\right)=\rho(a),
$$

which shows that each $\rho \in \Gamma$ is multibroadcastable by $\widetilde{K}_{*}$.

Remark 1 An interesting observation obtained from the proof of Theorem 5 is that the stronger assumption of complete positivity of a (dual) channel gives in fact the same set of multibroadcastable states. Indeed, if $\Gamma$ is multibroadcastable by a Schwarz channel, then the channel $K_{*}$ as defined in the proof of the implication (ii) $\Longrightarrow$ (i) in the theorem has completely positive dual and multibroadcasts the states from $\Gamma$.

Let us get rid now of the assumption of the faithfulness of $\Gamma$. Put

$$
p=\bigvee_{\rho \in \Gamma} \mathrm{s}(\rho)
$$

and consider the $W^{*}$-algebra $p \mathcal{A} p$. Then $\Gamma$ is a faithful family of normal states on $p \mathcal{A} p$. Now the idea of further considerations lies in 'transferring' the setup to the algebra $p \mathcal{A} p$, using the ergodic theorem for $W^{*}$-algebras there, and then coming back to the algebra $\mathcal{A}$. In particular, Theorem 5 holds in this more general setting. For the details of this procedure, the reader is referred to [7]. 
Let $K_{*}$ be a channel. Denote by $\mathcal{B}\left(K_{*}\right)$ the set of all normal states on $\mathcal{A}$ multibroadcast by $K_{*}$. Let, as before, $\mathfrak{S}$ be the semigroup generated by $L$ and $R$. Then a state $\rho$ belongs to $\mathcal{B}\left(K_{*}\right)$ if and only if it is $\mathfrak{S}$-invariant. Assume that $\mathcal{B}\left(K_{*}\right)$ is non-void. Then by the ergodic theorem for $W^{*}$-algebras there exists a normal unital projection $\mathbb{E}$ from $\mathcal{A}$ onto $\mathcal{F}(\mathfrak{S})$, and we have

$$
\mathcal{B}\left(K_{*}\right)=\{\rho-\text { normal state }: \rho \circ \mathbb{E}=\rho\} .
$$

(If $\mathcal{B}\left(K_{*}\right)$ is a faithful family, then $\mathbb{E}$ is a conditional expectation. If $\mathcal{B}\left(K_{*}\right)$ is not faithful, then $\mathcal{F}(\mathfrak{S})$ is still a $W^{*}$-algebra but with identity $p$ defined as

$$
p=\bigvee_{\rho \in \mathcal{B}\left(K_{*}\right)} \mathrm{s}(\rho)
$$

and $\mathbb{E}$ is a unital projection, i.e. $\mathbb{E}\left(\mathbb{1}_{\mathcal{A}}\right)=p$; see [7] for more details.) Moreover, putting $\Gamma=\mathcal{B}\left(K_{*}\right)$ in Theorem 5, we obtain the following description of $\mathcal{B}\left(K_{*}\right)$.

Corollary 6 There exists a family $\left\{\omega_{i}: i \in I\right\}$ of normal states with pairwise orthogonal supports such that

$$
\mathcal{B}\left(K_{*}\right)=\left\{\sum_{i \in I} \lambda_{i} \omega_{i}: \lambda_{i} \geq 0, \sum_{i \in I} \lambda_{i}=1\right\} .
$$

Finally, consider 1-to- $n$ multibroadcasting. Assume that for a channel $K_{*}$ and some fixed $j$ and $k$, the relations

$$
\rho\left(K\left(\mathbb{1}_{\mathcal{A}} \otimes \cdots \otimes \mathbb{1}_{\mathcal{A}} \otimes \underset{j}{a} \otimes \mathbb{1}_{\mathcal{A}} \otimes \cdots \otimes \mathbb{1}_{\mathcal{A}}\right)\right)=\rho(a), \quad a \in \mathcal{A},
$$

and

$$
\rho\left(K\left(\mathbb{1}_{\mathcal{A}} \otimes \cdots \otimes \mathbb{1}_{\mathcal{A}} \otimes \underset{k}{a} \otimes \mathbb{1}_{\mathcal{A}} \otimes \cdots \otimes \mathbb{1}_{\mathcal{A}}\right)\right)=\rho(a), \quad a \in \mathcal{A},
$$

hold for all $\rho \in \Gamma$. Call it two-broadcasting of $\rho$. Denote by $T_{i}$ the maps on $\mathcal{A}$ defined as

$$
T_{i}(a)=K\left(\mathbb{1}_{\mathcal{A}} \otimes \cdots \otimes \mathbb{1}_{\mathcal{A}} \otimes \underset{i}{a} \otimes \mathbb{1}_{\mathcal{A}} \otimes \cdots \otimes \mathbb{1}_{\mathcal{A}}\right) .
$$

According to our considerations in the Introduction, if the relations

$$
\rho \circ T_{i}=\rho
$$

hold for all $i=1, \ldots, n$, we speak about multibroadcasting of $\rho$. The following counterparts of Lemma 1 and Proposition 2 hold true.

Lemma $1^{\prime}$ For each $a \in \mathcal{F}\left(T_{i}\right)$, we have

$$
\mathbb{1}_{\mathcal{A}} \otimes \cdots \otimes \mathbb{1}_{\mathcal{A}} \otimes \underset{i}{a} \otimes \mathbb{1}_{\mathcal{A}} \otimes \cdots \otimes \mathbb{1}_{\mathcal{A}} \in \mathcal{N}
$$

Proposition $2^{\prime}$ Let $\mathfrak{S}$ be the semigroup generated by the maps $T_{j}$ and $T_{k}$. Then for any $x, y \in \mathcal{F}(\mathfrak{S})$, we have

$$
K\left(\mathbb{1}_{\mathcal{A}} \otimes \cdots \otimes \mathbb{1}_{\mathcal{A}} \otimes \underset{j}{x} \otimes \mathbb{1}_{\mathcal{A}} \otimes \cdots \otimes \mathbb{1}_{\mathcal{A}} \otimes \underset{k}{y} \otimes \mathbb{1}_{\mathcal{A}} \otimes \cdots \otimes \mathbb{1}_{\mathcal{A}}\right)=x y
$$

moreover, the von Neumann algebra $\mathcal{F}(\mathfrak{S})$ is abelian.

Proofs of these results are virtually the same as the proofs of Lemma 1 and Proposition 2.

Having at our disposal Lemma $1^{\prime}$ and Proposition $2^{\prime}$ above, we can show the following counterpart of Proposition 3. 
Proposition $3^{\prime}$ Let $\mathcal{M}$ be an abelian von Neumann algebra. The following conditions are equivalent:

(i) there exists a bounded normal Schwarz map $\widetilde{K}: \mathcal{N} \bar{\otimes} \ldots \bar{\otimes} \mathcal{M} \rightarrow \mathcal{M}$ satisfying for all $x, y \in \mathcal{M}$ the relation

$$
\widetilde{K}\left(\mathbb{1}_{\mathcal{M}} \otimes \cdots \otimes \mathbb{1}_{\mathcal{M}} \otimes \underset{j}{x} \otimes \mathbb{1}_{\mathcal{M}} \otimes \cdots \otimes \mathbb{1}_{\mathcal{M}} \otimes \underset{k}{y} \otimes \mathbb{1}_{\mathcal{M}} \otimes \cdots \otimes \mathbb{1}_{\mathcal{M}}\right)=x y,
$$

(ii) $\mathcal{M}$ is atomic, i.e. generated by the minimal projections.

The proof is again almost the same as the proof of Proposition 3.

As a corollary we get that the fixed-point algebra $\mathcal{F}(\mathfrak{S})$ is atomic and abelian. Following the lines of proof of Theorem 5 implication (i) $\Longrightarrow$ (ii), we obtain that the states in $\Gamma$ have the form of convex combinations of some $\omega_{i}$ with orthogonal supports.

Conversely, if the states in $\Gamma$ are convex combinations of states $\omega_{i}$ with orthogonal supports $e_{i}$, then defining a map

$$
\widetilde{K}_{*}: \mathcal{A}_{*} \rightarrow(\mathcal{A} \bar{\otimes} \ldots \bar{\otimes} \mathcal{A})_{*}
$$

by

$$
\widetilde{K}_{*} \varphi=\sum_{i} \varphi\left(e_{i}\right) \omega_{i} \otimes \cdots \otimes \omega_{i}, \quad \varphi \in \mathcal{A}_{*},
$$

we easily check that all $\rho$ in $\Gamma$ are multibroadcast by $K_{*}$, i.e. for each $i=1, \ldots, n$, we have

$$
\rho\left(K\left(\mathbb{1}_{\mathcal{A}} \otimes \cdots \otimes \mathbb{1}_{\mathcal{A}} \otimes \underset{i}{a} \otimes \mathbb{1}_{\mathcal{A}} \otimes \cdots \otimes \mathbb{1}_{\mathcal{A}}\right)\right)=\rho(a) .
$$

Thus we have shown that 1-to- $n$ multibroadcasting is equivalent to two-broadcasting.

\section{Multicloning}

In this section, we aim at obtaining a characterization of multicloneability of an arbitrary set of normal states $\Gamma$. As before, we shall assume without loss of generality that the states in $\Gamma$ form a faithful family.

Theorem 7 Let $\Gamma$ be an arbitrary faithful family of normal states on a von Neumann algebra $\mathcal{A}$. The following conditions are equivalent:

(i) $\Gamma$ is multicloneable,

(ii) the states in $\Gamma$ have pairwise orthogonal supports.

Proof (i) $\Longrightarrow$ (ii). Let $K_{*}: \mathcal{A}_{*} \rightarrow(\mathcal{A} \bar{\otimes} \ldots \bar{\otimes} \mathcal{A})_{*}$ multiclones the states in $\Gamma$. We shall adopt the setup and notation of Section 3. For each $\rho \in \Gamma$, we have $K_{*} \rho=\rho \otimes \cdots \otimes \rho$, so taking into account Proposition $2^{\prime}$, we obtain the equality

$$
\begin{aligned}
& \rho(x) \rho(y)=\rho \otimes \cdots \otimes \rho\left(\mathbb{1}_{\mathcal{A}} \otimes \cdots \otimes x \otimes \cdots \otimes y \otimes \cdots \otimes \mathbb{1}_{\mathcal{A}}\right) \\
& =\left(K_{*} \rho\right)\left(\mathbb{1}_{\mathcal{A}} \otimes \cdots \otimes \underset{j}{x} \otimes \cdots \otimes \underset{k}{y} \otimes \cdots \otimes \mathbb{1}_{\mathcal{A}}\right) \\
& =\rho\left(K\left(\mathbb{1}_{\mathcal{A}} \otimes \cdots \otimes \underset{j}{x} \otimes \cdots \otimes \underset{k}{y} \otimes \cdots \otimes \mathbb{1}_{\mathcal{A}}\right)\right)=\rho(x y)
\end{aligned}
$$

for all $x, y \in \mathcal{F}(\mathfrak{S})$. The equality above yields that for each projection $e \in \mathcal{F}(\mathfrak{S})$, and any $\rho \in \Gamma$, we have

$$
\rho(e)=0 \text { or } 1 .
$$


Since $\rho$, being cloned by $K_{*}$, is obviously also broadcast by $K_{*}$, it is $\mathfrak{S}$-invariant, so by virtue of [13, Lemma 1], $s(\rho) \in \mathcal{F}(\mathfrak{S})$; moreover, $s(\rho)$ is a minimal projection in $\mathcal{F}(\mathfrak{S})$. Indeed, for a projection $e \in \mathcal{F}(\mathfrak{S})$ such that $e \leq \mathrm{s}(\rho)$ and $e \neq \mathrm{s}(\rho)$, we cannot have $\rho(e)=1$, thus $\rho(e)=0$, and the faithfulness of $\rho$ on the algebra $\mathrm{s}(\rho) \mathcal{A} \mathrm{s}(\rho)$ yields $e=0$.

On account of Theorem $5, \mathcal{F}(\mathfrak{S})$ is an abelian atomic algebra generated by minimal projections $e_{i}$, moreover, the $e_{i}$ are the only minimal projections in $\mathcal{F}(\mathfrak{S})$. Thus, for each $\rho \in \Gamma$, we have s $(\rho)=e_{j}$ for some $e_{j}$. Now, if $\rho$ and $\varphi$ are distinct states in $\Gamma$, then their supports being minimal must be either orthogonal or equal. But if $\mathrm{s}(\rho)=\mathrm{s}(\varphi)=e_{j}$, then we would have

$$
\rho\left(\sum_{i} \alpha_{i} e_{i}\right)=\alpha_{j}
$$

and

$$
\varphi\left(\sum_{i} \alpha_{i} e_{i}\right)=\alpha_{j}
$$

showing that

$$
\rho|\mathcal{F}(\mathfrak{S})=\varphi| \mathcal{F}(\mathfrak{S})
$$

Let $\mathbb{E}$ be the conditional expectation onto $\mathcal{F}(\mathfrak{S})$ defined by means of the ergodic theorem. We have $\rho=\rho \circ \mathbb{E}$ and $\varphi=\varphi \circ \mathbb{E}$, thus equality (10) yields $\rho=\varphi$, contrary to the assumption that $\rho$ and $\varphi$ are distinct. Consequently, $\rho$ and $\varphi$ have orthogonal supports.

(ii) $\Longrightarrow$ (i). Denote $\Gamma=\left\{\omega_{i}\right\}$, and let $e_{i}$ be the support of $\omega_{i}$. Define the map $\widetilde{K}: \mathcal{A}_{*} \rightarrow$ $(\mathcal{A} \bar{\otimes} \ldots \bar{\otimes} \mathcal{A})_{*}$ as before, i.e.

$$
\widetilde{K}_{*} \varphi=\sum_{i} \varphi\left(e_{i}\right) \omega_{i} \otimes \cdots \otimes \omega_{i}, \quad \varphi \in \mathcal{A}_{*} .
$$

Then it is immediate that $\widetilde{K}$ multiclones all the $\omega_{i}$.

Suppose that a faithful family of normal states $\Gamma$ is multicloned by channel $K_{*}$, and denote as before by $\mathcal{B}\left(K_{*}\right)$ the set of all normal states on $\mathcal{A}$ multibroadcast by $K_{*}$. Then obviously $\Gamma \subset \mathcal{B}\left(K_{*}\right)$. However, we have more.

Proposition 8 All states in $\Gamma$ are extreme points of $\mathcal{B}\left(K_{*}\right)$.

Proof Take arbitrary $\rho \in \Gamma$. Equality (9) shows that $\rho \mid \mathcal{F}(\mathfrak{S})$ is a character of the abelian algebra $\mathcal{F}(\mathfrak{S})$, thus it is a pure state on this algebra, which means that it is an extreme point of the set of all states of this algebra. Now, if we have

$$
\rho=\lambda \varphi_{1}+(1-\lambda) \varphi_{2}
$$

for some $0<\lambda<1$ and $\varphi_{1}, \varphi_{2} \in \mathcal{B}\left(K_{*}\right)$, then

$$
\rho\left|\mathcal{F}(\mathfrak{S})=\lambda \varphi_{1}\right| \mathcal{F}(\mathfrak{S})+(1-\lambda) \varphi_{2} \mid \mathcal{F}(\mathfrak{S}),
$$

yielding the relation

$$
\rho\left|\mathcal{F}(\mathfrak{S})=\varphi_{1}\right| \mathcal{F}(\mathfrak{S})=\varphi_{2} \mid \mathcal{F}(\mathfrak{S}) .
$$

From relation (7), it follows that each state in $\mathcal{B}\left(K_{*}\right)$ is $\mathbb{E}$-invariant, thus we get for each $a \in \mathcal{A}$

$$
\rho(a)=\rho(\mathbb{E} a)=\varphi_{1,2}(\mathbb{E} a)=\varphi_{1,2}(a),
$$

showing that $\rho$ is an extreme point of $\mathcal{B}\left(K_{*}\right)$. 
Finally, let us consider the uniqueness of the cloning operation. For this we need yet another version of Proposition 2.

Proposition $2^{\prime \prime}$ Let $\Gamma$ be an arbitrary faithful family of normal states on a von Neumann algebra $\mathcal{A}$, and let $K_{*}$ be a channel broadcasting the states of $\Gamma$. Let the maps $T_{i}$ be defined by formula (8), and let $\mathfrak{S}$ be the semigroup generated by all $T_{i}$ ' $s$. Then for any $x_{1}, \ldots, x_{n} \in$ $\mathcal{F}(\mathfrak{S})$, we have

$$
K\left(x_{1} \otimes \cdots \otimes x_{n}\right)=x_{1} \ldots x_{n}
$$

Proof We have

$$
\mathcal{F}(\mathfrak{S})=\bigcap_{i=1}^{n} \mathcal{F}\left(T_{i}\right),
$$

so for any $x_{1}, \ldots, x_{n} \in \mathcal{F}(\mathfrak{S})$, and $i=1, \ldots, n$, Lemma $1^{\prime}$ yields

$$
\mathbb{1}_{\mathcal{A}} \otimes \cdots \otimes \mathbb{1}_{\mathcal{A}} \otimes x_{i} \otimes \mathbb{1}_{\mathcal{A}} \otimes \cdots \otimes \mathbb{1}_{\mathcal{A}} \in \mathcal{N} .
$$

Now, the following equality holds

$$
x_{1} \otimes \cdots \otimes x_{n}=\prod_{i=1}^{n}\left(\mathbb{1}_{\mathcal{A}} \otimes \cdots \otimes \mathbb{1}_{\mathcal{A}} \otimes x_{i} \otimes \mathbb{1}_{\mathcal{A}} \otimes \cdots \otimes \mathbb{1}_{\mathcal{A}}\right),
$$

hence from the homomorphic nature of $K$ on $\mathcal{N}$, we obtain

$$
\begin{aligned}
K\left(x_{1} \otimes \cdots \otimes x_{n}\right) & =\prod_{i=1}^{n} K\left(\mathbb{1}_{\mathcal{A}} \otimes \cdots \otimes \mathbb{1}_{\mathcal{A}} \otimes x_{i} \otimes \mathbb{1}_{\mathcal{A}} \otimes \cdots \otimes \mathbb{1}_{\mathcal{A}}\right) \\
& =\prod_{i=1}^{n} T_{i}\left(x_{i}\right)=x_{1} \ldots x_{n} .
\end{aligned}
$$

Theorem 9 Let $\Gamma=\left\{\rho_{i}\right\}$ be an arbitrary faithful family of normal states on a von Neumann algebra $\mathcal{A}$ such that the states in $\Gamma$ have pairwise orthogonal supports. Put $e_{i}=\mathrm{s}\left(\rho_{i}\right)$, and define the cloning channel $\hat{K}_{*}$ as before, i.e.

$$
\hat{K}_{*} \varphi=\sum_{i} \varphi\left(e_{i}\right) \rho_{i} \otimes \cdots \otimes \rho_{i}, \quad \varphi \in \mathcal{A}_{*} .
$$

Then for each channel $K_{*}$ that clones $\Gamma$, we have

$$
\hat{K}_{*}=(\mathbb{E} \otimes \cdots \otimes \mathbb{E})_{*} K_{*},
$$

where $\mathbb{E}$ is the conditional expectation from $\mathcal{A}$ onto $\mathcal{F}(\mathfrak{S})$ defined by means of the ergodic theorem as in Section 3.

Proof Let $K_{*}$ be a channel cloning $\Gamma$. Proposition 8 asserts that the $\rho_{i}$ are extreme points of $\mathcal{B}\left(K_{*}\right)$. From the description of $\mathcal{B}\left(K_{*}\right)$ obtained in Corollary 6, we know that the states $\omega_{i}$ as defined there form the set of all extreme points of $\mathcal{B}\left(K_{*}\right)$, thus we have $\left\{\rho_{i}\right\} \subset\left\{\omega_{i}\right\}$. Since

$$
\mathbb{1}=\sum_{i} e_{i}=\sum_{i} \mathrm{~s}\left(\rho_{i}\right) \leq \sum_{i} \mathrm{~s}\left(\omega_{i}\right) \leq \mathbb{1},
$$


we infer that $\left\{\rho_{i}\right\}=\left\{\omega_{i}\right\}$, and we may clearly assume that $\rho_{i}=\omega_{i}$ for each $i$. Consequently,

$$
\hat{K}_{*} \varphi=\sum_{i} \varphi\left(e_{i}\right) \omega_{i} \otimes \cdots \otimes \omega_{i}, \quad \varphi \in \mathcal{A}_{*} .
$$

In particular, for $a_{1}, \ldots, a_{n} \in \mathcal{A}$ we have, denoting by $\hat{K}$ the dual of $\hat{K}_{*}$,

$$
\hat{K}\left(a_{1} \otimes \cdots \otimes a_{n}\right)=\sum_{i} \omega_{i}\left(a_{1}\right) \ldots \omega_{i}\left(a_{n}\right) e_{i}
$$

For arbitrary normal state $\varphi$ on $\mathcal{A}$, relation (7) yields $\varphi \circ \mathbb{E} \in \mathcal{B}\left(K_{*}\right)$, thus on account of Corollary 6 we have

$$
\varphi \circ \mathbb{E}=\sum_{i} \lambda_{i}(\varphi) \omega_{i}
$$

where $\lambda_{i}(\varphi)$ are coefficients depending on $\varphi$. Since the $e_{i}$ are the supports of E-invariant states $\omega_{i}$, we have $\mathbb{E} e_{i}=e_{i}$, consequently,

$$
\varphi\left(e_{j}\right)=\varphi\left(\mathbb{E} e_{j}\right)=\lambda_{j}(\varphi)
$$

so

$$
\varphi \circ \mathbb{E}=\sum_{i} \varphi\left(e_{i}\right) \omega_{i}
$$

Hence for arbitrary $a \in \mathcal{A}$ and normal state $\varphi$ on $\mathcal{A}$, we obtain

$$
\varphi(\mathbb{E} a)=\sum_{i} \varphi\left(e_{i}\right) \omega_{i}(a),
$$

which means that

$$
\mathbb{E} a=\sum_{i} \omega_{i}(a) e_{i}
$$

This yields the formula

$$
\mathbb{E} a_{1} \ldots \mathbb{E} a_{n}=\sum_{i} \omega_{i}\left(a_{1}\right) \ldots \omega_{i}\left(a_{n}\right) e_{i}
$$

for all $a_{1}, \ldots, a_{n} \in \mathcal{A}$.

On account of Proposition $2^{\prime \prime}$, the following equality holds

$$
\begin{aligned}
K\left(\mathbb{E} \otimes \cdots \otimes \mathbb{E}\left(a_{1} \otimes \cdots \otimes a_{n}\right)\right) & =K\left(\mathbb{E} a_{1} \otimes \cdots \otimes \mathbb{E} a_{n}\right) \\
& =\mathbb{E} a_{1} \cdots \mathbb{E} a_{n} .
\end{aligned}
$$

Formulas (11), (12) and (13) yield

$$
\hat{K}\left(a_{1} \otimes \cdots \otimes a_{n}\right)=K\left(\mathbb{E} \otimes \cdots \otimes \mathbb{E}\left(a_{1} \otimes \cdots \otimes a_{n}\right)\right),
$$

for any $a_{1}, \ldots a_{n} \in \mathcal{A}$, which means that

$$
\hat{K}=K(\mathbb{E} \otimes \cdots \otimes \mathbb{E}),
$$

and thus

$$
\hat{K}_{*}=(\mathbb{E} \otimes \cdots \otimes \mathbb{E})_{*} K_{*},
$$

finishing the proof.

Remark 2 It is probably worth noting that the assumption of faithfulness of $\Gamma$ in the above theorem is essential. 


\section{Concluding Remarks}

Finally, we would like to briefly comment on the general $m$-to- $n$ broadcasting problem for arbitrary $m<n$. The simplest case is $m=2, n=3$. Then we look for a channel

$$
K_{*}:(\mathcal{A} \bar{\otimes} \mathcal{A})_{*} \rightarrow(\mathcal{A} \bar{\otimes} \mathcal{A} \bar{\otimes} \mathcal{A})_{*}
$$

such that for a state $\tilde{\rho} \in(\mathcal{A} \bar{\otimes} \mathcal{A})_{*}$ to be broadcast, we should have

$$
\tilde{\rho}\left(K\left(\widetilde{a} \otimes \mathbb{1}_{\mathcal{A}}\right)\right)=\widetilde{\rho}\left(K\left(\mathbb{1}_{\mathcal{A}} \otimes \widetilde{a}\right)\right)=\widetilde{\rho}(\widetilde{a}) \quad \text { for each } \quad \widetilde{a} \in \mathcal{A} \bar{\otimes} \mathcal{A} .
$$

It is surprising that even for one state $\widetilde{\rho}$ only, giving an example of such $K_{*}$ creates problems unless $\widetilde{\rho}$ is of the form $\rho \otimes \rho$, in which case we can take e.g.

$$
K_{*} \tilde{\varphi}=\widetilde{\varphi}\left(\mathbb{1}_{\mathcal{A}} \otimes \mathbb{1}_{\mathcal{A}}\right) \rho \otimes \rho \otimes \rho, \quad \widetilde{\varphi} \in(\mathcal{A} \bar{\otimes} \mathcal{A})_{*} .
$$

The reason for this is the following. We still have $\mathcal{A} \bar{\otimes} \mathcal{A}$ as the left and right marginals of $\mathcal{A} \bar{\otimes} \mathcal{A} \bar{\otimes} \mathcal{A}$, but now these marginals are not independent, i.e. commuting, as was the case for the algebra $\mathcal{A} \bar{\otimes} \mathcal{B} \overline{\mathcal{A}}$ where the marginals $\mathcal{A}$ were identified with $\mathcal{A} \bar{\otimes} \mathbb{1}_{\mathcal{B}} \bar{\otimes} \mathbb{1}_{\mathcal{A}}$ and $\mathbb{1}_{\mathcal{A}} \bar{\otimes} \mathbb{1}_{\mathcal{B}} \bar{\otimes} \mathcal{A}$, respectively. Consequently, our methods fail, and the question of multibroadcasting in this case remains open.

Open Access This article is distributed under the terms of the Creative Commons Attribution License which permits any use, distribution, and reproduction in any medium, provided the original author(s) and the source are credited.

\section{References}

1. Barnum, H., Barrett, J., Leifer, M., Wilce, A.: Cloning and broadcasting in generic probability models. preprint, arXiv:quant-ph/0611295

2. Barnum, H., Barrett, J., Leifer, M., Wilce, A.: Generalized no-broadcasting theorem. Phys. Rev. Lett. 99, 240501 (2007)

3. Barnum, H., Caves, C.M., Fuchs, C.A., Jozsa, R., Schumacher, B.: Noncommuting mixed states cannot be broadcast, Phys. Rev. Lett. 76(15), 2818-2821 (1996)

4. Dang, G.-F., Fan, H.: Optimal broadcasting of mixed states. Phys. Rev. A 76, 022323 (2007)

5. D’Ariano, G.M., Macchiavello, C., Perinotti, P.: Superbroadcasting of mixed states. Phys. Rev. Lett. 95(6), 060503 (2005)

6. Dieks, D.: Communication by EPR devices. Phys. Lett. A 92(6), 271-272 (1982)

7. Kaniowski, K., Lubnauer, K., Łuczak, A.: Cloning and broadcasting in operator algebras, to appear in Quart. J. Math.

8. Kaniowski, K., Lubnauer, K., Łuczak, A.: Cloning in $C^{*}$-algebras, preprint

9. Kümmerer, B., Nagel, R.: Mean ergodic semigroups on $W^{*}$-algebras. Acta Sci. Math. (Szeged) 41, 151159 (1979)

10. Lindblad, G.: A general no-cloning theorem. Lett. Math. Phys. 47, 189-196 (1999)

11. Łuczak, A.: Cloning by positive maps in von Neumann algebras, to appear in Positivity.

12. Scarani, V., Iblisdir, S., Gisin, N., Acin, A.: Quantum cloning. Rev. Modern Phys. 77(4), 1225 (2005)

13. Thomsen, K.E.: Invariant states for operator semigroups. Studia Math. 81, 285-291 (1985)

14. Wooters, W.K., Zurek, W.H.: A single quantum state cannot be cloned. Nature 299, 802 (1982) 